Proyecciones Journal of Mathematics

Vol. 36, $\mathrm{N}^{\circ}$ 2, pp. 325-346, June 2017.

Universidad Católica del Norte

Antofagasta - Chile

\title{
On some spaces of Lacunary I-convergent sequences of interval numbers defined by sequence of moduli
}

\author{
Mohd Shafiq \\ Govt. Degree College, India \\ and \\ Ayhan Esi \\ Adiyaman University, Turkey \\ Received: October 2016. Accepted : January 2017
}

\begin{abstract}
In this article we introduce and study some spaces of I-convergent sequences of interval numbers with the help of a sequence $\mathcal{F}=\left(f_{k}\right)$ of moduli, a bounded sequence $p=\left(p_{k}\right)$ of positive real numbers and a lacunary sequence $\theta=\left\{k_{r}\right\}$ of increasing integers. We study some topological and algebraic properties and some inclusion relations on these spaces.
\end{abstract}

Keywords and Phrases : Interval numbers, Ideal, filter, I-convergent sequence, solid and monotone space, Banach space, modulus function. 


\section{Introduction and Preliminaries}

Let $\mathbf{N}, \mathbf{R}$ and $\mathbf{C}$ be the sets of all natural, real and complex numbers, respectively.

Let $\ell_{\infty}, c$ and $c_{0}$ be denote the Banach spaces of bounded, convergent and null sequences, respectively with norm

$$
\|x\|=\sup _{k}\left|x_{k}\right|
$$

We denote

$$
\omega=\left\{x=\left(x_{k}\right): x_{k} \in \mathbf{R o r} \mathbf{C}\right\}
$$

the space of all real or complex sequences.

It is an admitted fact that the real and complex numbers are playing a vital role in the world of mathematics. Many mathematical structures have been constructed with the help of these numbers. In recent years, since 1965 fuzzy numbers and interval numbers also managed their place in the world of mathematics and credited into account some alike structures . Interval arithmetic was first suggested by P.S.Dwyer [12] in 1951. Further development of interval arithmetic as a formal system and evidence of its value as a computational device was provided by R.E.Moore [22] in 1959 and Moore and Yang [23] and others and have developed applications to differential equations.

Recently, Chiao [11] introduced sequences of interval numbers and defined usual convergence of sequences of interval numbers. Şengönül and Eryllmaz [34] introduced and studied bounded and convergent sequence spaces of interval numbers and showed that these spaces are complete.

A set (closed interval) of real numbers $x$ such that $a \leq x \leq b$ is called an interval number [11]. A real interval can also be considered as a set. Thus, we can investigate some properties of interval numbers for instance, arithmetic properties or analysis properties. Let us denote the set of all real valued closed intervals by $I \mathbf{R}$. Any element of $I \mathbf{R}$ is called a closed interval and it is denoted by $\bar{A}=\left[x_{l}, x_{r}\right] . I \mathbf{R}$ is a quasilinear space under the algebraic operations and a partial order relation for $I \mathbf{R}$ found in [34] and any subspace of $I \mathbf{R}$ is called quasilinear subspace (see [34]). Further research on interval numbers was carried out by Esi and Braha [6], Esi and Hazarika [8], Esi [1-9] and the references therein.

The set of all interval numbers $I \mathbf{R}$ is a complete metric space defined by 


$$
d\left(\bar{A}_{1}, \bar{A}_{2}\right)=\max \left\{\left|x_{1_{l}}-x_{2_{l}}\right|,\left|x_{1_{r}}-x_{2_{r}}\right|\right\},(\sec [23,34]) .
$$

where $x_{l}$ and $x_{r}$ be first and last points of $\bar{A}$, respectively.

In a special case, $\bar{A}_{1}=[a, a], \bar{A}_{2}=[b, b]$, we obtain the usual metric of $\mathbf{R}$ with

$$
d\left(\bar{A}_{1}, \bar{A}_{2}\right)=|a-b| .
$$

Let us define transformation $f$ from $\mathbf{N}$ to $I \mathbf{R}$ by $k \rightarrow f(k)=\widehat{A}, \mathbb{A}=$ $\left(\overline{A_{k}}\right)$. The function $f$ is called sequence of interval numbers, where $\overline{A_{k}}$ is the $k^{\text {th }}$ term of the sequence $\left(\bar{A}_{k}\right)$.

Let us denote the set of sequences of interval numbers with real terms by

$$
\omega(\widehat{\mathcal{A}})=\left\{\widehat{\mathcal{A}}=\left(\overline{A_{k}}\right): \overline{A_{k}} \in I \mathbf{R}\right\} .
$$

The algebraic properties of $\omega(\mathcal{A})$ can be found in $[11,34]$.

The following definitions were given by Şengönül and Eryılmaz in [34].

A sequence $\widehat{A}=\left(\overline{A_{k}}\right)=\left(\left[x_{k_{l}}, x_{k_{r}}\right]\right)$ of interval numbers is said to be convergent to an interval number $\bar{A}_{0}=\left[x_{0_{l}}, x_{0_{r}}\right]$ if for each $\epsilon>0$, there exists a positive integer $n_{0}$ such that $d\left(\bar{A}_{k}, \bar{A}_{0}\right)<\epsilon$, for all $k \geq n_{0}$ and we denote it as $\lim _{k} \overline{A_{k}}=\overline{A_{0}}$.

Thus, $\lim _{k} \overline{A_{k}}=\overline{A_{0}} \Leftrightarrow \lim _{k} x_{k_{l}}=x_{0_{l}}$ and $\lim _{k} x_{k_{r}}=x_{0_{r}}$ and it is said to be Cauchy sequence of interval numbers if for each $\epsilon>0$, there exists a positive integer $k_{0}$ such that $d\left(\overline{A_{k}}, \overline{A_{m}}\right)<\epsilon$, whenever $k, m \geq k_{0}$.

Let us denote the space of all convergent, null and bounded sequences of interval numbers by $\mathcal{C}(\mathcal{A}), \mathcal{C}_{\circ}(\mathcal{A})$ and $\ell_{\infty}(\mathcal{A})$, respectively. The sets $\mathcal{C}(\mathcal{A})$, $\mathcal{C}_{\circ}(\mathcal{A})$ and $\ell_{\infty}(\mathcal{A})$ are complete metric spaces with the metric

$$
\widehat{d}\left(\bar{A}_{k}, \bar{B}_{k}\right)=\sup _{k} \max \left\{\left|x_{k_{l}}-y_{k_{l}}\right|,\left|x_{k_{r}}-y_{k_{r}}\right|\right\}(\operatorname{see}[34]) .
$$


If we take $\bar{B}_{k}=\overline{0}$ in (3) then, the metric $\widehat{d}$ reduces to

$$
\widehat{d}\left(\bar{A}_{k}, \overline{0}\right)=\sup _{k} \max \left\{\left|x_{k_{l}}\right|,\left|x_{k_{r}}\right|\right\} .
$$

In this paper, we assume that a norm $\left\|\bar{A}_{k}\right\|$ of the sequence of interval numbers $\left(\bar{A}_{k}\right)$ is the distance from $\left(\bar{A}_{k}\right)$ to $\overline{0}$ and satisfies the following properties:

$$
\begin{aligned}
& \forall \bar{A}_{k}, \bar{B}_{k} \in \lambda(\bar{A}) \text { and } \forall \alpha \in \mathbf{R} \\
& \left(N_{1}\right) \forall \bar{A}_{k} \in \lambda(\widetilde{\mathcal{A}})-\{\overline{0}\},\left\|\overline{A_{k}}\right\|_{\lambda(\text { 氶 })}>0, \\
& \left(N_{2}\right)\left\|\bar{A}_{k}\right\|_{\lambda(\mathcal{A})}=0 \Leftrightarrow \bar{A}_{k}=\overline{0},
\end{aligned}
$$

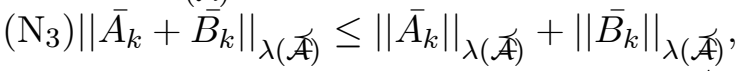

$$
\begin{aligned}
& \left.\left(N_{4}\right)|| \alpha \overline{A_{k}}\right|_{\lambda(\mathcal{A})}=|\alpha| \|\left.\bar{A}_{k}\right|_{\lambda(\widehat{A})} \text {, where } \lambda(\widehat{A}) \text { is a subset of } \omega(\widehat{A}) \text {. }
\end{aligned}
$$

Let $\widehat{\mathcal{A}}=\left(\overline{A_{k}}\right)=\left(\left[x_{k_{l}}, x_{k_{r}}\right]\right)$ be the element of $\mathcal{C}(\widehat{\mathcal{A}}), \mathcal{C}_{\circ}(\mathcal{A})$ or $\ell_{\infty}(\widehat{A})$. Then, in the light of above discussion, the classes $\mathcal{C}(\mathcal{A}), \mathcal{C}_{\circ}(\mathcal{A})$ and $\ell_{\infty}(\mathcal{A})$ of sequences of interval numbers are normed interval spaces normed by

$$
\| \text { 本 } \|=\sup _{k} \max \left\{\left|x_{k_{l}}\right|,\left|x_{k_{r}}\right|\right\}(\text { see[34]). }
$$

Throughout, $\overline{0}=[0,0]$ and $\bar{I}=[1,1]$ represent zero and identity interval numbers according to addition and multiplication, respectively.

As a generalisation of usual convergence for the sequences of real or complex numbers, the concept of statistical convergent was first introduced by Fast [13] and also independently by Buck [10] and Schoenberg [33]. Later on, it was further investigated from a sequence space point of view and linked with the Summability Theory by Fridy [15], Šalát [30], Tripathy [35] and many others. The statistical convergence has been extended to interval numbers by Esi and others as follows in [1-9].

Let us suppose that $\widehat{\mathcal{A}}=\left(\bar{A}_{k}\right) \in \ell_{\infty}(\mathcal{A})$. If, for every $\epsilon>0$,

$$
\lim _{n} \frac{1}{n}\left|\left\{k \in \mathbf{N}:\left\|\bar{A}_{k}-\bar{A}_{0}\right\| \geq \epsilon, k \leq n\right\}\right|=0 .
$$

Then, the sequence $\widehat{A}=\left(\bar{A}_{k}\right)$ is said to be statistically convergent to an interval number $\bar{A}_{0}$, where vertical lines denote the cardinality of the enclosed set. That is, if $\delta(A(\epsilon))=0$, where $A(\epsilon)=\left\{k \in \mathbf{N}:\left\|\bar{A}_{k}-\bar{A}_{0}\right\| \geq \epsilon\right\}$.

The notion of ideal convergence (I-convergence) was introduced and studied by Kostyrko, Mačaj, Salăt and Wilczyński [20, 21]. Later on, it 
was studied by Šalát, Tripathy and Ziman [31, 32], Esi and Hazarika [8], Tripathy and Hazarika [36], Khan etal [16, 17], Mursaleen and Sunil [25] and many others.

Definition 1.1. Let $\mathbf{N}$ be the set of natural numbers. Then, a family of sets $I \subseteq 2^{\mathbf{N}}$ (power set of $\mathbf{N}$ ) is said to be an ideal, if

(i) $I$ is additive. That is, $\forall A, B \in I \Rightarrow A \cup B \in I$,

(ii) $I$ is hereditary. That is $\forall A \in \operatorname{Iand} B \subseteq A \Rightarrow B \in I$.

$A$ non-empty family of sets $£(I) \subseteq 2^{\mathbf{N}}$ is said to be filter on $\mathbf{N}$, if and only if

(i) $\Phi \notin £(I)$,

(ii) $\forall A, B \in £(I)$ we have $A \cap B \in £(I)$,

(iii) $\forall A \in £(I)$ and $A \subseteq B \Rightarrow B \in £(I)$.

An Ideal $I \subseteq 2^{\mathbf{N}}$ is called non-trivial if $I \neq 2^{\mathbf{N}}$.

A non-trivial ideal $I \subseteq 2^{\mathbf{N}}$ is called admissible if

$$
\{\{x\}: x \in \mathbf{N}\} \subseteq I \text {. }
$$

Let us suppose that $I$ be an ideal. Then, a sequence $\mathbb{A}=\left(\bar{A}_{k}\right) \in \ell_{\infty}(\mathcal{A}) \subset$ $\omega($ A)

(i) is said to be I-convergent to an interval number $\bar{A}_{0}$ if for every $\epsilon>0$,

$$
\left\{k \in \mathbf{N}:\left\|\bar{A}_{k}-\bar{A}_{0}\right\| \geq \epsilon\right\} \in I .
$$

In this case, we write $I-\lim \bar{A}_{k}=\overline{A_{0}}$. If $\overline{A_{0}}=\bar{O}$.

Then, the sequence $\widehat{A}=\left(\bar{A}_{k}\right) \in \ell_{\infty}(\widehat{A})$ is said to be $I$-null. In this case, we write $I-\lim \bar{A}_{k}=\bar{O}$.

(ii) is said to be I-Cauchy, if for every $\epsilon>0$, there exists a number $m=$ $m(\epsilon)$ such that

$$
\left\{k \in \mathbf{N}:\left\|\overline{A_{k}}-\overline{A_{m}}\right\| \geq \epsilon\right\} \in I .
$$

(iii) is said to be I-bounded, if there exists some $M>0$ such that

$$
\left\{k \in \mathbf{N}:\left\|\bar{A}_{k}\right\| \geq M\right\} \in I .
$$

We know that for each ideal $I$, there is a filter $£(I)$ corresponding to $I$. That is, $£(I)=\left\{K \subseteq \mathbf{N}: K^{c} \in I\right\}$, where $K^{c}=\mathbf{N} \backslash K$.

Definition 1.2. A sequence space $\lambda(\widehat{A})$ of interval numbers is (iv) said to be solid(normal), if $\left(\alpha_{k} \bar{A}_{k}\right) \in \lambda(\mathcal{A})$, whenever $\left(\bar{A}_{k}\right) \in \lambda(\mathcal{A})$ and for any sequence $\left(\alpha_{k}\right)$ of scalars with $\left|\alpha_{k}\right| \leq 1$, for all $k \in \mathbf{N}$,

(v) said to be symmetric, if $\left(\bar{A}_{\pi(k)}\right) \in \lambda(\mathcal{A})$, whenever $\bar{A}_{k} \in \lambda(\mathcal{A})$, where $\pi$ 
is a permutation on $\mathbf{N}$,

(vi) said to be sequence algebra, if $\left(\bar{A}_{k}\right) *\left(\bar{B}_{k}\right)=\left(\bar{A}_{k} \cdot \bar{B}_{k}\right) \in \lambda($ A) whenever $\left(\bar{A}_{k}\right),\left(\bar{B}_{k}\right) \in \lambda(\mathcal{A})$,

(vii) said to be convergence free, if $\left(\bar{B}_{k}\right) \in \lambda(\widehat{A})$ whenever $\left(\bar{A}_{k}\right) \in \lambda(\mathcal{A})$ and $\bar{A}_{k}=\bar{O}$ implies $\bar{B}_{k}=\bar{O}$, for all $k$.

Definition 1.3. Let $K=\left\{k_{1}<k_{2}<k_{3} \ldots\right\} \subset \mathbf{N}$. The $K$-step space of the

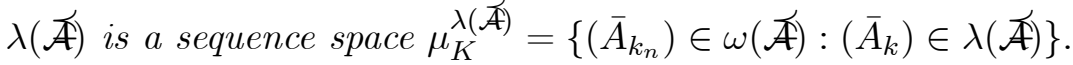

Definition 1.4. A canonical pre-image of a sequence $\left(\bar{A}_{k_{n}}\right) \in \mu_{K}^{\lambda(\mathcal{A})}$ is a sequence

$\left(\bar{B}_{k}\right) \in \omega(\mathcal{A})$ defined by

$$
\bar{B}_{k}=\left\{\begin{array}{l}
\bar{A}_{k}, \text { if } k \in K \\
\bar{O}, \text { otherwise }
\end{array}\right.
$$

A canonical preimage of a step space $\mu_{K}^{\lambda(\mathcal{A})}$ is a set of canonical preimages of all elements in $\mu_{K}^{\lambda(\sqrt{\mathcal{A}})}$. That is, $\widehat{\mathcal{F}}$ is in the canonical preimage of $\mu_{K}^{\lambda(\mathcal{A})}$ iff $\widehat{\mathcal{B}}$ is the canonical preimage of some $\widehat{\mathcal{A}} \in \mu_{K}^{\lambda(\mathcal{A})}$.

Definition 1.5. A sequence space $\lambda(\widehat{A})$ is said to be monotone, if it contains the canonical preimages of its step space.

Definition 1.6. A function $f:[0, \infty) \longrightarrow[0, \infty)$ is called a modulus function if

(1) $f(t)=0$ if and only if $t=0$,

$f(t+u) \leq f(t)+f(u)$ for all $t, u \geq 0$,

(3) $f$ is increasing, and

(4) $f$ is continuous from the right at zero.

$A$ modulus function $f$ is said to satisfy $\Delta_{2}$ - Condition for all values of $u$ if there exists a constant $K>0$ such that $f(L u) \leq K L f(u)$ for all values of $L>1$.

The idea of modulus function was introduced by Nakano in 1953, (See [26], Nakano, 1953).

Ruckle [27-29] used the idea of a modulus function $f$ to construct the sequence space

$\left(1.7 X(f)=\left\{x=\left(x_{k}\right): \sum_{k=1}^{\infty} f\left(\left|x_{k}\right|\right)<\infty\right\}=\left\{x=x_{k}:\left(f\left(\left|x_{k}\right|\right)\right) \in X\right\}\right.$. 
After then, E.Kolk [18-19] gave an extension of $X(f)$ by considering a sequence of modulii $\mathcal{F}=\left(f_{k}\right)$ and defined the sequence space

$$
X(\mathcal{F})=\left\{x=\left(x_{k}\right):\left(f_{k}\left(\left|x_{k}\right|\right)\right) \in X\right\} .
$$

Mursaleen and Noman [24] introduced the notion of $\lambda$-convergent and $\lambda$ bounded sequences. We extended this concept to the sequence of interval numbers as follows.

Let $\lambda=\left(\lambda_{k}\right)_{k=1}^{\infty}$ be a strictly increasing sequence of positive real numbers tending to infinity. That is,

$$
0<\lambda_{0}<\lambda_{1}<\lambda_{2}<\lambda_{3} \ldots, \lambda_{k} \rightarrow \infty \text { ask } \rightarrow \infty
$$

The sequence $\widehat{A}=\left(\bar{A}_{k}\right) \in \ell_{\infty}(\widehat{A})$ is $\lambda$-convergent to an interval number $\overline{A_{0}}$, called the $\lambda$-limit of $\widehat{\mathcal{A}}$, if $\Lambda_{m}(\widetilde{\mathcal{A}}) \rightarrow \overline{A_{0}}$, as $m \rightarrow \infty$, where

$$
\Lambda_{m}(\text { 氶 })=\frac{1}{\lambda_{m}} \sum_{k=1}^{m}\left(\lambda_{k}-\lambda_{k-1}\right) \bar{A}_{k},(k \in \mathbf{N}) .
$$

Here and in the sequel, we shall use the convention that any term with a negative subscript is equal to naught. For example, $\lambda_{-1}=0$.

In particular, $\widehat{\mathcal{A}}=\left(\bar{A}_{k}\right) \in \ell_{\infty}(\mathcal{A})$ is said to be $\lambda$-null, if $\Lambda_{m}(\mathcal{A}) \rightarrow 0$, as $m \rightarrow \infty$.

The sequence 氶 $=\left(\bar{A}_{k}\right) \in \ell_{\infty}\left(\right.$ 氶) is $\lambda$-bounded, if $\sup _{m} \| \Lambda_{m}($ 氶 $) \|<\infty$. It can be seen that if $\lim _{m} \bar{A}_{m}=\bar{A}$ in the ordinary sense of convergence of interval numbers, then

$$
\lim _{m}\left(\frac{1}{\lambda_{m}}\left(\sum_{k=1}^{m}\left(\lambda_{k}-\lambda_{k-1}\right)\left\|\bar{A}_{k}-\bar{A}\right\|\right)\right)=0 .
$$

This implies that

(1.11) $\lim _{m} \| \Lambda_{m}\left(\right.$ 承 $-\bar{A}\left\|=\lim _{m}\right\| \frac{1}{\lambda_{m}} \sum_{k=1}^{m}\left(\lambda_{k}-\lambda_{k-1}\right)\left(\bar{A}_{k}-\bar{A}\right) \|=0$.

which yields that $\lim _{m} \Lambda_{m}(\widehat{\mathcal{A}})=\bar{A}$ and hence $\widehat{A}=\left(\bar{A}_{k}\right) \in \ell_{\infty}($ 正 $)$ is $\lambda$ convergent to $\bar{A}$. 
Let us denote the classes of I-convergent, I-null, bounded I-convergent and bounded I-null sequences of interval numbers with $\mathcal{C}^{I}(\widehat{A}), \mathcal{C}_{\circ}^{I}(\mathcal{A}), \mathcal{M}_{\mathcal{C}}^{I}(\mathbb{A})$ and $\mathcal{M}_{\mathcal{C}_{0}}^{I}(\mathcal{A})$, respectively.

By a lacunary sequence we mean an increasing integer sequence $\theta=\left\{k_{r}\right\}$ such that $k_{0}=0$ and $h_{r}:=k_{r}-k_{r-1} \rightarrow \infty$ as $r \rightarrow \infty$. Throughout this paper the intervals determined by $\theta$ will be denoted by $I_{r}:=\left(k_{r-1}, k_{r}\right)$ and the ratio $\frac{k_{r}}{k_{r-1}}$ will be abbreviated by $q_{r}$

The space of lacunary strongly convergent sequence $N_{\theta}$ was defined by Freedman etal[14] as:

$$
N_{\theta}=\left\{x=x_{k}: \lim _{r} \frac{1}{h_{r}} \sum_{k \in I_{r}}\left|x_{k}-\ell\right| \text { for some }\right\}
$$

We need the following popular inequalities throughout the paper.

Let $p=\left(p_{k}\right)$ be the bounded sequence of positive reals numbers. For any complex $\lambda$, whenever $H=\sup _{k}\left(p_{k}\right)<\infty$, we have

$$
|\lambda|^{p_{k}} \leq \max \left(1,|\lambda|^{H}\right)
$$

Also, whenever $H=\sup _{k}\left(p_{k}\right)$, we have

$$
\left|a_{k}+b_{k}\right|^{p_{k}} \leq C\left(\left|a_{k}\right|^{p_{k}}+\left|b_{k}\right|^{p_{k}}\right) .
$$

where $D=\max \left(1,2^{H-1}\right)$.

For any modulus function $f$, we have the inequalities

$$
|f(x)-f(y)| \leq f(x-y)
$$

and

$$
f(n x) \leq n f(x), \text { for allx }, y \in[0, \infty] .
$$

Now, we give some important Lemmas.

Lemma.1.7. Every solid space is monotone.

Lemma.1.8. Let $K \in £(I)$ and $M \subseteq \mathbf{N}$. If $M \notin I$, then $M \cap K \notin I$, where $£(I) \subseteq 2^{\mathbf{N}}$ filter on $\mathbf{N}$.

Lemma.1.9. If $I \subseteq 2^{\mathbf{N}}$ and $M \subseteq \mathbf{N}$. If $M \notin I$, then $M \cap \mathbf{N} \notin I$.

Let us give a most important definitions for this paper. 
Definition 1.10 (see [37]). Let $\bar{X}$ be a quasilinear space of interval numbers. A function $g: \bar{X} \longrightarrow \mathbf{R}$ is called paranorm on $\bar{X}$, if for all $A, B \in \bar{X}$, $\left(P_{1}\right) g(A)=0$ if $A=\overline{0}$,

$\left(P_{2}\right) g(A) \geq 0$,

$\left(P_{3}\right) g(-A)=g(A)$

$\left(P_{4}\right) g(A+B) \leq g(A)+g(B)$,

$\left(P_{5}\right)$ if $\left(\lambda_{n}\right)$ is a sequence of scalars with $\lambda_{n} \rightarrow \lambda(n \rightarrow \infty)$ and $\left(A_{n}\right), A_{0} \in \bar{X}$

with $g\left(A_{n}\right) \rightarrow g\left(A_{0}\right)(n \rightarrow \infty)$, then $g\left(\lambda_{n} A_{n}\right) \rightarrow g\left(\lambda A_{0}\right)(n \rightarrow \infty)$,

$\left(P_{6}\right)$ If $A \leq B$, then $g(A) \leq g(B)$.

Definition 1.11 (see [37]). Suppose that $X$ is a quasilinear space and $Y \subset X$. $Y$ is called a subspace of $X$ whenever $Y$ is a quasilinear space with the same partial ordering and same operations on $X$.

Theorem 1.12 (see [37]). $Y$ is a subspace of a quasilinear space $X$ if and only if for every $x, y \in Y$ and $\alpha, \beta \in \mathbf{R}, \alpha x+\beta y \in Y$.

In this article, we introduce and study the following classes of sequences;

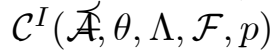

$$
\begin{aligned}
=\{\widehat{\mathcal{A}} & \left.=\left(\bar{A}_{k}\right) \in \ell_{\infty}(\widehat{\mathcal{A}}):\left\{r \in \mathbf{N}: \frac{1}{h_{r}} \sum_{k \in I_{r}} f_{k}\left(\left\|\Lambda_{k}(\widehat{\mathcal{A}})-\bar{A}\right\|\right)^{p_{k}} \geq \epsilon\right\} \in I, \text { for some } \bar{A}\right\} \\
& (1.12) \\
& \mathcal{C}_{0}^{I}(\widehat{\mathcal{A}}, \theta, \Lambda, \mathcal{F}, p) \\
& =\left\{\widehat{\mathcal{A}}=\left(\bar{A}_{k}\right) \in \ell_{\infty}(\widehat{\mathcal{A}}):\left\{r \in \mathbf{N}: \frac{1}{h_{r}} \sum_{k \in I_{r}} f_{k}\left(\left\|\Lambda_{k}(\widehat{\mathcal{A}})\right\|\right)^{p_{k}} \geq \epsilon\right\} \in I\right\} ;
\end{aligned}
$$

$$
\begin{aligned}
& \ell_{\infty}^{I}(\widehat{\mathcal{A}}, \theta, \Lambda, \mathcal{F}, p) \\
= & \left\{\widehat{\mathcal{A}}=\left(\bar{A}_{k}\right) \in \ell_{\infty}(\widehat{\mathcal{A}}): \exists K>0 \text { s.t. }\left\{r \in \mathbf{N}: \frac{1}{h_{r}} \sum_{k_{r}} f_{k}\left(\| \Lambda_{k}(\text { 承 } \|)^{p_{k}} \geq K\right\} \in I\right\} ;\right.
\end{aligned}
$$




$$
\begin{aligned}
& \ell_{\infty}(\widehat{\mathcal{A}}, \theta, \Lambda, \mathcal{F}, p) \\
& (1.15)=\left\{\widehat{\mathcal{A}}=\left(\bar{A}_{k}\right) \in \ell_{\infty}(\text { 氶 }): \sup _{r} \frac{1}{h_{r}} \sum_{k \in I_{r}} f_{k}\left(\| \Lambda_{k}(\text { 舟 } \|)^{p_{k}}<\infty\right\} .\right.
\end{aligned}
$$

We also denote

$$
\mathcal{M}_{\mathcal{C}}^{I}(\widehat{\mathcal{A}}, \theta, \Lambda, \mathcal{F}, p)=\ell_{\infty}(\widehat{\mathcal{A}}, \theta, \Lambda, \mathcal{F}, p) \cap \mathcal{C}^{I}(\widehat{A}, \theta, \Lambda, \mathcal{F}, p)
$$

and

$\mathcal{M}_{\mathcal{C}_{\circ}}^{I}(\mathcal{A}, \theta, \Lambda, \mathcal{F}, p)=\ell_{\infty}(\mathcal{A}, \theta, \Lambda, \mathcal{F}, p) \cap \mathcal{C}_{0}^{I}(\widehat{\mathcal{A}}, \theta . \Lambda, \mathcal{F}, p)$, where $p=\left(p_{k}\right)$ is a bounded sequence of positive real numbers, $\mathcal{F}=\left(f_{k}\right)$ is a sequence of modulus functions, $\theta=\left\{k_{r}\right\}$ is a lacunary sequence and $\widehat{A}=\left(\bar{A}_{k}\right) \in$ $\ell_{\infty}(\widehat{A}) \subset \omega(\widehat{A})$ is a bounded sequence of interval numbers.

\section{Main Results}

Theorem 2.1. Let $\mathcal{F}=\left(f_{k}\right)$ be a sequence of modulus functions and $p=\left(p_{k}\right)$ be the bounded sequence of positive real numbers. Then, the sets $\mathcal{C}^{I}(\widehat{A}, \theta, \Lambda, \mathcal{F}, p), \mathcal{C}_{0}^{I}(\widehat{A}, \theta, \Lambda, \mathcal{F}, p), \mathcal{M}_{\mathcal{C}}^{I}(\widehat{A}, \theta, \Lambda, \mathcal{F}, p)$ and $\mathcal{M}_{\mathcal{C}_{\circ}}^{I}(\widehat{A}, \theta, \Lambda, \mathcal{F}, p)$ are quasilinear spaces over the field of real numbers.

Proof. We shall prove the result for the space $\mathcal{C}^{I}(\mathcal{A}, \theta, \Lambda, \mathcal{F}, p)$. Rests will follow similarly.

For, let $\widehat{\mathcal{A}}=\left(\bar{A}_{k}\right), \widetilde{\mathcal{B}}=\left(\bar{B}_{k}\right) \in \mathcal{C}^{I}(\widetilde{\mathcal{A}}, \theta, \Lambda, \mathcal{F}, p)$ and $\alpha, \beta$ be scalars. Then, there exist positive integers $M_{\alpha}$ and $N_{\beta}$ such that $|\alpha| \leq M_{\alpha}$ and $|\beta| \leq N_{\beta}$. Now, since $\widehat{\mathbb{A}}=\left(\bar{A}_{k}\right), \widetilde{\mathcal{F}}=\left(\bar{B}_{k}\right) \in \mathcal{C}^{I}(\widehat{\mathcal{A}}, \theta, \Lambda, \mathcal{F}, p)$, then, there exists $\bar{A}, \bar{B} \in I \mathbf{R}$ such that the sets

$$
A_{1}=\left\{r \in \mathbf{N}: \frac{1}{h_{r}} \sum_{k \in I_{r}} f_{k}\left(\left\|\Lambda_{k}(\overline{\mathcal{A}})-\bar{A}\right\|\right)^{p_{k}} \geq \epsilon\right\} \in I
$$

and

$$
A_{2}=\left\{r \in \mathbf{N}: \frac{1}{h_{r}} \sum_{k \in I_{r}} f_{k}\left(\left\|\Lambda_{k}(\widetilde{\mathcal{B}})-\bar{B}\right\|\right)^{p_{k}} \geq \epsilon\right\} \in I .
$$

Since, each $f_{k}, k \in \mathbf{N}$ is modulus function, we have,

$$
\frac{1}{h_{r}} \sum_{k \in I_{r}} f_{k}\left(\left\|\Lambda_{k}(\alpha \widehat{\mathcal{A}}+\beta \widetilde{\mathcal{B}})-(\alpha \bar{A}+\beta \bar{A})\right\|\right)^{p_{k}}
$$




$$
\begin{aligned}
& \leq D\left(M_{\alpha}\right)^{H} \times \frac{1}{h_{r}} \sum_{k \in I_{r}} f_{k}\left(\left\|\Lambda_{k}(\widehat{\mathcal{A}})-\bar{A}\right\|\right)^{p_{k}} \\
& +D\left(N_{\beta}\right)^{H} \times \frac{1}{h_{r}} \sum_{k \in I_{r}} f_{k}\left(\left\|\Lambda_{k}(\widetilde{\mathcal{B}})-\bar{B}\right\|\right)^{p_{k}}
\end{aligned}
$$

The above inequality further implies that

$$
\begin{gathered}
\left\{r \in \mathbf{N}: \frac{1}{h_{r}} \sum_{k \in I_{r}} f_{k}\left(\left\|\Lambda_{k}(\alpha \widehat{\mathcal{A}}+\beta \widetilde{\mathcal{B}})-(\alpha \bar{A}+\beta \bar{A})\right\|\right)^{p_{k}} \geq \epsilon\right\} \\
\subseteq\left\{r \in \mathbf{N}: \frac{1}{h_{r}} \sum_{k \in I_{r}} f_{k}\left(\left\|\Lambda_{k}(\widehat{\mathcal{A}})-\bar{A}\right\|\right)^{p_{k}} \geq \epsilon\right\} \\
\cup\left\{r \in \mathbf{N}: \frac{1}{h_{r}} \sum_{k \in I_{r}} f_{k}\left(\left\|\Lambda_{k}(\widetilde{\mathcal{B}})-\bar{B}\right\|\right)^{p_{k}} \geq \epsilon\right\} \\
=A_{2} \cup A_{2} \in I
\end{gathered}
$$

The result follows from the above inclusion and the definition of quasilinear space.

Theorem 2.2. Let $\mathcal{F}=\left(f_{k}\right)$ be a sequence of modulus functions and $p=$ $\left(p_{k}\right)$ be the bounded sequence of positive real numbers. Then, the classes of sequences $\mathcal{M}_{\mathcal{C}}^{I}(\widehat{\mathcal{A}}, \theta, \Lambda, \mathcal{F}, p)$ and $\mathcal{M}_{\mathcal{C}_{\circ}}^{I}(\widehat{\mathcal{A}}, \theta, \Lambda, \mathcal{F}, p)$ are paranormed spaces, paranormed by

$g(\mathcal{A})=g\left(\left(\bar{A}_{k}\right)\right)=\sup _{r} \frac{1}{h_{r}} \sum_{k \in I_{r}} f_{k}\left(\left\|\bar{\Lambda}_{k}\left(A_{k}\right)\right\|\right)^{\frac{p_{k}}{M}}$, where $M=\max \left\{1, \sup _{k} p_{k}\right\}$.

Proof. Let $\widehat{\mathcal{A}}=\left(\bar{A}_{k}\right), \widetilde{\mathcal{B}}=\left(\bar{B}_{k}\right) \in \mathcal{M}_{\mathcal{C}}^{I}(\widehat{A}, \theta, \Lambda, \mathcal{F}, p)$.

$\left(P_{1}\right)$ It is Clear that $g(\widehat{A})=0$, if $\widehat{A}=\bar{\theta}$.

$\left(P_{2}\right)$ It is also obvious that $g(\widehat{A}) \geq 0$.

$\left(P_{3}\right) g(\mathcal{A})=g(-\mathcal{A})$ is obvious.

$\left(P_{4}\right)$ Since $\frac{p_{k}}{M} \leq 1$ and $M>1$, using Minkowski's inequality, we have

$$
g(\widehat{\mathcal{A}}+\widetilde{\mathcal{B}})=g\left(\overline{A_{k}}+\overline{B_{k}}\right)
$$




$$
\begin{gathered}
\left.=\sup _{r} \frac{1}{h_{r}} \sum_{k \in I_{r}} f_{k}\left(\| \Lambda_{k}\left(\bar{A}_{k}+\bar{B}_{k}\right)\right) \|\right)^{\frac{p_{k}}{M}} \\
\left.=\sup _{r} \frac{1}{h_{r}} \sum_{k \in I_{r}} f_{k}\left(\| \Lambda_{k}\left(\bar{A}_{k}\right)+\Lambda_{k}\left(\bar{B}_{k}\right)\right) \|\right)^{\frac{p_{k}}{M}} \\
\left.\leq \sup _{r} \frac{1}{h_{r}} \sum_{k \in I_{r}} f_{k}\left(\left\|\Lambda_{k}\left(\bar{A}_{k}\right)\right\|^{\frac{p_{k}}{M}}+\| \Lambda_{k}\left(\bar{B}_{k}\right)\right) \|\right)^{\frac{p_{k}}{M}} \\
\leq \sup _{r} \frac{1}{h_{r}} \sum_{k \in I_{r}} f_{k}\left(\left\|\Lambda_{k}\left(\bar{A}_{k}\right)\right\|\right)^{\frac{p_{k}}{M}} \\
+\sup _{r} \frac{1}{h_{r}} \sum_{k \in I_{r}} f_{k}\left(\| \Lambda_{k}\left(\bar{B}_{k}\right)\right) \|^{\frac{p_{k}}{M}} \\
=g(\widetilde{\mathcal{A}})+g(\widetilde{\mathcal{B}}) .
\end{gathered}
$$

Therefore, $g(\widehat{\mathbb{A}}+\widetilde{\mathcal{B}}) \leq g(\widehat{\mathbb{A}})+g(\widetilde{\mathcal{B}})$, for all $\widehat{\mathcal{A}}, \widetilde{\mathcal{B}} \in \mathcal{M}_{\mathcal{C}}^{I}(\widehat{\mathcal{A}}, \theta, \Lambda, \mathcal{F}, p)$. $\left(P_{5}\right)$ Let $\left(\lambda_{k}\right)$ be a sequence of scalars with $\left(\lambda_{k}\right) \rightarrow \lambda(k \rightarrow \infty)$ and $\left(\bar{A}_{k}\right), \bar{A}_{0} \in \mathcal{M}_{\mathcal{C}}^{I}(\overline{\mathcal{A}}, \theta, \Lambda, \mathcal{F}, p)$ such that

$$
\bar{A}_{k} \rightarrow \bar{A}_{0}(k \rightarrow \infty)
$$

in the sense that

$$
g\left(\bar{A}_{k}-\bar{A}_{0}\right) \rightarrow 0(k \rightarrow \infty) .
$$

Note that $g(\lambda$ 承 $\leq \max \{1,|\lambda|\} g$ (氶).

Then, since the inequality

$$
g\left(\bar{A}_{k}\right) \leq g\left(\bar{A}_{k}-\bar{A}_{0}\right)+g\left(\bar{A}_{0}\right)
$$

holds by subadditivity of $g$, the sequence $\left\{g\left(\bar{A}_{k}\right)\right\}$ is bounded.

Therefore,

$$
\begin{gathered}
\left|g\left(\lambda_{k} \bar{A}_{k}\right)-g\left(\lambda \bar{A}_{0}\right)\right|=\left|g\left(\lambda_{k} \bar{A}_{k}\right)-g\left(\lambda \bar{A}_{k}\right)+g\left(\lambda \bar{A}_{k}\right)-g\left(\lambda \bar{A}_{0}\right)\right| \\
\leq\left|\lambda_{k}-\lambda\right|^{\frac{p_{k}}{M}}\left|g\left(\bar{A}_{k}\right)\right|+|\lambda|^{\frac{p_{k}}{M}}\left|g\left(\bar{A}_{k}\right)-g\left(\bar{A}_{0}\right)\right| \rightarrow 0
\end{gathered}
$$

as $(k \rightarrow \infty)$. That is to say that scalar multiplication is continuous. $\left(P_{6}\right)$. Since each $f_{k}, k \in \mathbf{N}$ is an increasing function, it is clear that $g(\mathcal{A}) \leq g(\mathbb{B})$, if $\mathcal{A} \subseteq \mathbb{B}$. 
Hence $\mathcal{M}_{\mathcal{C}}^{I}(\widehat{A}, \theta, \Lambda, \mathcal{F}, p)$ is a paranormed space.

For $\mathcal{M}_{\mathcal{C}_{\circ}}^{I}(\mathcal{A}, \theta, \Lambda, \mathcal{F}, p)$, the result is similar.

Theorem 2.3. The set $\mathcal{M}_{\mathcal{C}}^{I}(\widehat{A}, \theta, \Lambda, \mathcal{F}, p)$ is closed subspace of $\ell_{\infty}(\widehat{A}, \theta, \Lambda, \mathcal{F}, p)$.

Proof. Let $\widehat{A}^{(n)}=\left(\bar{A}_{k}^{(n)}\right)$ be a Cauchy sequence in $\mathcal{M}_{\mathcal{C}}^{I}(\widetilde{\mathcal{A}}, \theta, \Lambda, \mathcal{F}, p)$ such that

$$
\bar{A}_{k}^{(n)} \rightarrow \bar{A}
$$

We show that $\bar{A} \in \mathcal{M}_{\mathcal{C}}^{I}(\widehat{A}, \theta, \Lambda, \mathcal{F}, p)$.

Since $\widehat{A}^{(n)}=\left(\bar{A}_{k}^{(n)}\right) \in \mathcal{M}_{\mathcal{C}}^{I}(\widehat{\mathcal{A}}, \theta, \Lambda, \mathcal{F}, p)$. Then, there exists $\bar{A}_{n}$ such that

$$
\left\{r \in \mathbf{N}: \frac{1}{h_{r}} \sum_{k \in I_{r}} f_{k}\left(\left\|\Lambda_{k}\left(\widehat{A}^{(n)}\right)-\overline{A_{n}}\right\|\right)^{p_{k}} \geq \epsilon\right\} \in I
$$

We need to show that

(1) $\left(\overline{A_{n}}\right)$ converges to $\overline{A_{0}}$.

(2) If $U=\left\{r \in \mathbf{N}: \frac{1}{h_{r}} \sum_{k \in I_{r}} f_{k}\left(\left\|\Lambda_{k}(\widehat{A})-\overline{A_{0}}\right\|\right)^{p_{k}}<\epsilon\right\}$, then $U^{c} \in I$.

(1) Since $\bar{A}^{(n)}=\left(\bar{A}_{k}^{(n)}\right)$ is Cauchy sequence in $\mathcal{M}_{\mathcal{C}}^{I}(\mathcal{A}, \theta, \Lambda, \mathcal{F}, p) \Rightarrow$ for a given $\epsilon>0$, there exists $k_{0} \in \mathbf{N}$ such that

$$
\sup _{r} \frac{1}{h_{r}} \sum_{k \in I_{r}} f_{k}\left(\left\|\Lambda_{k}\left(\widehat{A}^{(n)}\right)-\Lambda_{k}\left(\widehat{A}^{(q)}\right)\right\|\right)^{\frac{p_{k}}{M}}<\frac{\epsilon}{3}, \text { for all } n, q \geq k_{0}
$$

where $M=\max \left\{1, \sup _{k} p_{k}\right\}$.

For $\epsilon>0$, we have

$$
\begin{gathered}
B_{n q}=\left\{r \in \mathbf{N}: \frac{1}{h_{r}} \sum_{k \in I_{r}} f_{k}\left(\left\|\Lambda_{k}\left(\widehat{A}^{(n)}\right)-\Lambda_{k}\left(\widehat{A}^{(q)}\right)\right\|\right)^{p_{k}}<\left(\frac{\epsilon}{3}\right)^{M}\right\}, \\
B_{q}=\left\{r \in \mathbf{N}: \frac{1}{h_{r}} \sum_{k \in I_{r}} f_{k}\left(\left\|\Lambda_{k}\left(\widehat{A}^{(q)}\right)-\bar{A}_{q}\right\|\right)^{p_{k}}<\left(\frac{\epsilon}{3}\right)^{M}\right\}, \\
B_{n}=\left\{r \in \mathbf{N}: \frac{1}{h_{r}} \sum_{k \in I_{r}} f_{k}\left(\left\|\Lambda_{k}\left(\widehat{A}^{(n)}\right)-\bar{A}_{n}\right\|\right)^{p_{k}}<\left(\frac{\epsilon}{3}\right)^{M}\right\} .
\end{gathered}
$$

Then, $B_{n q}^{c}, B_{q}^{c}, B_{n}^{c} \in I$ 
Let $B^{c}=B_{n q}^{c} \cup B_{q}^{c} \cup B_{n}^{c}$, where

$$
B=\left\{r \in \mathbf{N}: \frac{1}{h_{r}} \sum_{k \in I_{r}} f_{k}\left(\left\|\overline{A_{q}}-\overline{A_{n}}\right\|\right)^{p_{k}}<\epsilon\right\} .
$$

Then, $B^{c} \in I$.

We choose $k_{0} \in B^{c}$. Then, for each $n, q \geq k_{0}$, we have $\left\{r \in \mathbf{N}: \frac{1}{h_{r}} \sum_{k \in I_{r}} f_{k}\left(\left\|\overline{A_{q}}-\overline{A_{n}}\right\|\right)^{p_{k}}<\epsilon\right\}$

$$
\begin{gathered}
\supseteq\left[\left\{r \in \mathbf{N}: \frac{1}{h_{r}} \sum_{k \in I_{r}} f_{k}\left(\left\|\bar{A}_{q}-\Lambda_{k}\left(\widehat{A}^{(q)}\right)\right\|\right)^{p_{k}}<\left(\frac{\epsilon}{3}\right)^{M}\right\}\right. \\
\cap\left\{r \in \mathbf{N}: \frac{1}{h_{r}} \sum_{k \in I_{r}} f_{k}\left(\left\|\Lambda_{k}\left(\widehat{A}^{(n)}\right)-\Lambda_{k}\left(\widehat{A}^{(q)}\right)\right\|\right)^{p_{k}}<\left(\frac{\epsilon}{3}\right)^{M}\right\} \\
\left.\cap\left\{r \in \mathbf{N}: \frac{1}{h_{r}} \sum_{k \in I_{r}} f_{k}\left(\left\|\Lambda_{k}\left(\widehat{\mathbb{A}}^{(n)}\right)-\overline{A_{n}}\right\|\right)^{p_{k}}<\left(\frac{\epsilon}{3}\right)^{M}\right\}\right] .
\end{gathered}
$$

Then, $\left(\overline{A_{n}}\right)$ is a Cauchy sequence of interval numbers, so there exists some interval number $\overline{A_{0}}$ such that $\overline{A_{n}} \rightarrow \overline{A_{0}}$ as $n \rightarrow \infty$.

(2) Let $0<\delta<1$ be given. Then, we show that, if

$$
U=\left\{r \in \mathbf{N}: \frac{1}{h_{r}} \sum_{k \in I_{r}} f_{k}\left(\left\|\Lambda_{k}(\widehat{\mathcal{A}})-\overline{A_{0}}\right\|\right)^{p_{k}}<\delta\right\},
$$

then, $U^{c} \in I$.

Since $\widehat{A}^{(n)}=\left(\bar{A}_{k}^{(n)}\right) \rightarrow \bar{A}$ then, there exists $q_{0} \in \mathbf{N}$ such that

$$
P=\left\{r \in \mathbf{N}: \frac{1}{h_{r}} \sum_{k \in I_{r}} f_{k}\left(\left\|\Lambda_{k}\left(\mathcal{A}^{\left(q_{0}\right)}\right)-\Lambda_{k}(\mathcal{A})\right\|\right)^{p_{k}}<\left(\frac{\delta}{3 D}\right)^{M}\right\}
$$

implies $P^{c} \in I$, where

$$
D=\max \left\{1,2^{H-1}\right\}, H=\sup _{k} p_{k} \geq 0 .
$$

The number $q_{0}$ can be chosen that together with (20), we have

$$
Q=\left\{r \in \mathbf{N}: \frac{1}{h_{r}} \sum_{k \in I_{r}} f_{k}\left(\left\|\Lambda_{k}\left(\bar{A}_{q_{0}}\right)-\bar{A}_{0}\right\|\right)^{p_{k}}<\left(\frac{\delta}{3 D}\right)^{M}\right\}
$$


such that $Q^{c} \in I$.

Since $\left\{r \in \mathbf{N}: \frac{1}{h_{r}} \sum_{k \in I_{r}} f_{k}\left(\left\|\Lambda_{k}\left(\widehat{A}^{\left(q_{0}\right)}\right)-\Lambda_{k}\left(\bar{A}_{q_{0}}\right)\right\|\right)^{p_{k}} \geq \delta\right\} \in I$. Then, we have a subset $S$ of $\mathbf{N}$ such that $S^{c} \in I$, where

$$
S=\left\{r \in \mathbf{N}: \frac{1}{h_{r}} \sum_{k \in I_{r}} f_{k}\left(\left\|\Lambda_{k}\left(\widehat{A}^{\left(q_{0}\right)}\right)-\Lambda_{k}\left(\bar{A}_{q_{0}}\right)\right\|\right)^{p_{k}}<\left(\frac{\delta}{3 D}\right)^{M}\right\} .
$$

Let $U^{c}=P^{c} \cup Q^{c} \cup S^{c}$, where

$$
U=\left\{r \in \mathbf{N}: \frac{1}{h_{r}} \sum_{k \in I_{r}} f_{k}\left(\left\|\Lambda_{k}(\mathcal{A})-\overline{A_{0}}\right\|\right)^{p_{k}}<\delta\right\} .
$$

Therefore, for each $k \in U^{c}$, we have

$$
\begin{aligned}
& \left\{r \in \mathbf{N}: \frac{1}{h_{r}} \sum_{k \in I_{r}} f_{k}\left(\left\|\Lambda_{k}(\overline{\mathcal{A}})-\bar{A}_{0}\right\|\right)^{p_{k}}<\delta\right\} \\
& \supseteq\left[\left\{r \in \mathbf{N}: \frac{1}{h_{r}} \sum_{k \in I_{r}} f_{k}\left(\left\|\Lambda_{k}\left(\overline{\mathcal{A}}^{\left(q_{0}\right)}\right)-\Lambda_{k}(\mathcal{A})\right\|\right)^{p_{k}}<\left(\frac{\delta}{3 D}\right)^{M}\right\}\right. \\
& \quad \cap\left\{r \in \mathbf{N}: \frac{1}{h_{r}} \sum_{k \in I_{r}} f_{k}\left(\left\|\Lambda_{k}\left(\bar{A}^{\left(q_{0}\right)}\right)-\Lambda_{k}\left(\bar{A}_{q_{0}}\right)\right\|\right)^{p_{k}}<\left(\frac{\delta}{3 D}\right)^{M}\right\} \\
& \left.(2.6) \quad \cap\left\{r \in \mathbf{N}: \frac{1}{h_{r}} \sum_{k \in I_{r}} f_{k}\left(\left\|\Lambda_{k}\left(\bar{A}_{q_{0}}\right)-\bar{A}_{0}\right\|\right)^{p_{k}}<\left(\frac{\delta}{3 D}\right)^{M}\right\}\right] .
\end{aligned}
$$

Then, the result follows from (21).

Since the inclusions $\mathcal{M}_{\mathcal{C}}^{I}(\widehat{\mathcal{A}}, \theta, \Lambda, \mathcal{F}, p) \subset \ell_{\infty}(\widehat{\mathcal{A}}, \theta, \Lambda, \mathcal{F}, p)$ and $\mathcal{M}_{\mathcal{C}_{\circ}}^{I}(\mathcal{A}, \theta, \Lambda, \mathcal{F}, p) \subset \ell_{\infty}(\mathcal{A}, \theta, \Lambda, \mathcal{F}, p)$ are strict so in view of Theorem $(2.3)$ we have the following result.

Theorem 2.4. The spaces $\mathcal{M}_{\mathcal{C}}^{I}(\widehat{\mathcal{A}}, \theta, \Lambda, \mathcal{F}, p)$ and $\mathcal{M}_{\mathcal{C}_{\circ}}^{I}(\widehat{A}, \theta, \Lambda, \mathcal{F}, p)$ are nowhere dense subsets of $\ell_{\infty}(\mathcal{A}, \theta, \Lambda, \mathcal{F}, p)$.

Theorem 2.5. The spaces $\mathcal{C}_{0}^{I}(\widehat{A}, \theta, \Lambda, \mathcal{F}, p)$ and $\mathcal{M}_{\mathcal{C}_{\circ}}^{I}(\widehat{A}, \theta, \Lambda, \mathcal{F}, p)$ are both solid and monotone.

Proof. We shall prove the result for $\mathcal{C}_{0}^{I}(\widehat{\mathcal{A}}, \theta, \Lambda, \mathcal{F}, p)$. For $\mathcal{M}_{\mathcal{C}_{\circ}}^{I}(\widehat{\mathcal{A}}, \theta, \Lambda, \mathcal{F}, p)$, the result follows similarly.

For, let $\widehat{\mathcal{A}}=\left(\bar{A}_{k}\right) \in \mathcal{C}_{0}^{I}(\widehat{A}, \theta, \Lambda, \mathcal{F}, p)$ and $\left(\alpha_{k}\right)$ be a sequence of scalars with $\left|\alpha_{k}\right| \leq 1$, for all $k \in \mathbf{N}$.

Since $\left|\alpha_{k}\right|^{p_{k}} \leq \max \left\{1,\left|\alpha_{k}\right|^{G}\right\} \leq 1$, for all $k \in \mathbf{N}$, we have 


$$
\frac{1}{h_{r}} \sum_{k \in I_{r}} f_{k}\left(\left\|\alpha_{k} \Lambda_{k}\left(\bar{A}_{k}\right)\right\|\right)^{p_{k}} \leq \frac{1}{h_{r}} \sum_{k \in I_{r}} f_{k}\left(\left\|\Lambda_{k}\left(\bar{A}_{k}\right)\right\|\right)^{p_{k}}, \text { for all } \mathrm{k} \in \mathbf{N} \text {. }
$$

which further implies that

$$
\begin{aligned}
& \left\{r \in \mathbf{N}: \frac{1}{h_{r}} \sum_{k \in I_{r}} f_{k}\left(\left\|\Lambda_{k}\left(\bar{A}_{k}\right)\right\|\right)^{p_{k}} \geq \epsilon\right\} \supseteq \\
& \left\{r \in \mathbf{N}: \frac{1}{h_{r}} \sum_{k \in I_{r}} f_{k}\left(\left\|\alpha_{k} \Lambda_{k}\left(\bar{A}_{k}\right)\right\|\right)^{p_{k}} \geq \epsilon\right\} .
\end{aligned}
$$

Thus, $\alpha_{k}\left(\bar{A}_{k}\right) \in \mathcal{C}_{0}^{I}(\widehat{A}, \theta, \Lambda, \mathcal{F}, p)$.

Therefore, the space $\mathcal{C}_{0}^{I}(\mathcal{A}, \theta, \Lambda, \mathcal{F}, p)$ is solid and hence by lemma (1.7), it is monotone.

Theorem 2.6. Let $\mathcal{F}=\left(f_{k}\right)$ and $\mathcal{G}=\left(g_{k}\right)$ be two sequences of modulus functions and for each $k \in \mathbf{N},\left(f_{k}\right)$ and $\left(g_{k}\right)$ satisfying $\Delta_{2}-$ Condition and $p=\left(p_{k}\right) \in \ell_{\infty}$ be a bounded sequence of positive real numbers. Then,

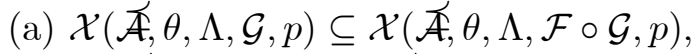

(b) $\mathcal{X}(\mathcal{A}, \theta, \Lambda, \mathcal{F}, p) \cap(\mathcal{A}, \theta, \Lambda, \mathcal{G}, p) \subseteq \mathcal{X}(\mathcal{A}, \theta, \Lambda, \mathcal{F}+\mathcal{G}, p)$,

for $\mathcal{X}=\mathcal{C}^{I}, \mathcal{C}_{\circ}^{I}, \mathcal{M}_{\mathcal{C}}^{I}$ and $\mathcal{M}_{\mathcal{C}_{\circ}}^{I}$

Proof. (a). Let $\widehat{A}=\left(\bar{A}_{k}\right) \in \mathcal{C}_{\circ}^{I}(\widehat{\mathcal{A}}, \theta, \Lambda, \mathcal{G}, p)$ be any element.

Then, the set

$$
\left\{r \in \mathbf{N}: \frac{1}{h_{r}} \sum_{k \in I_{r}} g_{k}\left(\left\|\Lambda_{k}\left(\bar{A}_{k}\right)\right\|\right)^{p_{k}} \geq \epsilon\right\} \in I .
$$

Let $\epsilon>0$ and choose $\delta$ with $0<\delta<1$ such that $f_{k}(t)<\epsilon, 0 \leq t \leq \delta$.

Let us denote

$$
\bar{B}_{k}=\frac{1}{h_{r}} \sum_{k \in I_{r}} g_{k}\left(\left\|\Lambda_{k}\left(\bar{A}_{k}\right)\right\|\right)^{p_{k}}
$$

and consider

$$
\lim _{k} f_{k}\left(\bar{B}_{k}\right)=\lim _{\bar{B}_{k} \leq \delta, k \in \mathbf{N}} f_{k}\left(\bar{B}_{k}\right)+\lim _{\bar{B}_{k}>\delta, k \in \mathbf{N}} f_{k}\left(\bar{B}_{k}\right) .
$$

Now, since $f_{k}$ for each $\mathrm{k} \in \mathbf{N}$ is an modulus function, we have

$$
\lim _{\bar{B}_{k} \leq \delta, k \in \mathbf{N}} f_{k}\left(\bar{B}_{k}\right) \leq f_{k}(2) \lim _{\bar{B}_{k} \leq \delta, k \in \mathbf{N}}\left(\bar{B}_{k}\right) .
$$


For $\bar{B}_{k}>\delta$, we have

$$
\bar{B}_{k}<\frac{\bar{B}_{k}}{\delta}<1+\frac{\bar{B}_{k}}{\delta} .
$$

Now, since each $f_{k}$ is non-decreasing and modulus, it follows that

$$
f_{k}\left(\bar{B}_{k}\right)<f_{k}\left(1+\frac{\bar{B}_{k}}{\delta}\right)<\frac{1}{2} f_{k}(2)+\frac{1}{2} f_{k}\left(\frac{2 \bar{B}_{k}}{\delta}\right) .
$$

Again, since each $f_{k}, k \in \mathbf{N}$ satisfies $\Delta_{2}$ - Condition, we have

$$
f_{k}\left(\bar{B}_{k}\right)<\frac{1}{2} K \frac{\left(\bar{B}_{k}\right)}{\delta} f_{k}(2)+\frac{1}{2} K \frac{\left(\bar{B}_{k}\right)}{\delta} f_{k}(2) .
$$

Thus, $f_{k}\left(\bar{B}_{k}\right)<K \frac{\left(\bar{B}_{k}\right)}{\delta} f_{k}(2)$.

Hence,

$\lim _{\bar{B}_{k}>\delta, k \in \mathbf{N}} f_{k}\left(\bar{B}_{k}\right) \leq \max \left\{1,\left(K \delta^{-1} f_{k}(2)\right)^{H}\right\} \lim _{\bar{B}_{k}>\delta, k \in \mathbf{N}}\left(\bar{B}_{k}\right), H=\max \left\{1, \sup _{k} p_{k}\right\}$.

$(2.10)$

Therefore, from (23), (24) and (25), we have

$\widehat{A}=\left(\bar{A}_{k}\right) \in \mathcal{C}_{\circ}^{I}(\widehat{A}, \theta, \Lambda, \mathcal{F} \circ \mathcal{G}, p)$.

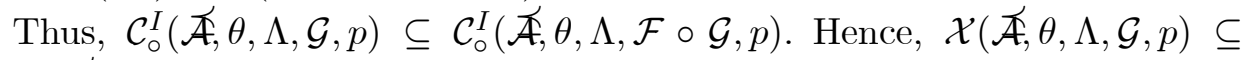
$\mathcal{X}(\mathcal{A}, \theta, \Lambda, \mathcal{F} \circ \mathcal{G}, p)$ for $\mathcal{X}=\mathcal{C}_{\circ}^{I}$.

For $\mathcal{X}=\mathcal{C}^{I}, \mathcal{M}_{\mathcal{C}}^{I}$ and $\mathcal{M}_{\mathcal{C}_{\circ}}^{I}$ the inclusions can be established similarly.

(b). Let $\widehat{\mathcal{A}}=\left(\bar{A}_{k}\right) \in \mathcal{C}_{\circ}^{I}(\mathcal{A}, \theta, \Lambda, \mathcal{F}, p) \cap \mathcal{C}_{\circ}^{I}(\mathcal{A}, \theta, \Lambda, \mathcal{G}, p)$. Let $\epsilon>0$ be given.

Then, the sets

$$
\left\{r \in \mathbf{N}: \frac{1}{h_{r}} \sum_{k \in I_{r}} f_{k}\left(\left\|\Lambda_{k}\left(\bar{A}_{k}\right)\right\|\right)^{p_{k}} \geq \epsilon\right\} \in I
$$

and

$$
\left\{r \in \mathbf{N}: \frac{1}{h_{r}} \sum_{k \in I_{r}} g_{k}\left(\left\|\Lambda_{k}\left(\bar{A}_{k}\right)\right\|\right)^{p_{k}} \geq \epsilon\right\} \in I .
$$

Therefore, from (26) and (27), we have

$$
\left\{r \in \mathbf{N}: \frac{1}{h_{r}} \sum_{k \in I_{r}}\left(f_{k}+g_{k}\right)\left(\left\|\Lambda_{k}\left(\bar{A}_{k}\right)\right\|\right)^{p_{k}} \geq \epsilon\right\} \in I .
$$

Thus, $\widehat{\mathcal{A}}=\left(\bar{A}_{k}\right) \in \mathcal{C}_{\circ}^{I}(\mathcal{A}, \theta, \Lambda, \mathcal{F}+\mathcal{G}, p)$ 
Hence, $\mathcal{C}_{\circ}^{I}(\widehat{A}, \theta, \Lambda, \mathcal{F}, p) \cap \mathcal{C}_{\circ}^{I}(\widehat{A}, \theta, \Lambda, \mathcal{G}, p) \subseteq \mathcal{C}_{\circ}^{I}(\widehat{A}, \theta, \Lambda, \mathcal{F}+\mathcal{G}, p)$.

For $\mathcal{X}=\mathcal{C}^{I}, \mathcal{M}_{\mathcal{C}}^{I}$ and $\mathcal{M}_{\mathcal{C}_{\circ}}^{I}$, the inclusions are similar.

For $g_{k}(x)=x$ and $f_{k}(x)=f(x), \forall x \in[0, \infty)$, we have the following corollary.

Corollary 2.7. $\mathcal{X}(\widehat{A}, \theta, \Lambda, p) \subseteq \mathcal{X}(\widehat{\mathcal{A}}, \theta, \Lambda, \mathcal{F}, p)$, for $\mathcal{X}=\mathcal{C}^{I}, \mathcal{C}_{\circ}^{I}, \mathcal{M}_{\mathcal{C}}^{I}$ and $\mathcal{M}_{\mathcal{C}_{\circ}}^{I}$

Theorem 2.8. Let $\mathcal{F}=\left(f_{k}\right)$ be a sequence of modulus functions. Then, the inclusions $\mathcal{C}_{0}^{I}(\widetilde{\mathcal{A}}, \theta, \Lambda, \mathcal{F}, p) \subset \mathcal{C}^{I}(\widehat{A}, \theta, \Lambda, \mathcal{F}, p) \subset \ell_{\infty}^{I}(\widehat{A}, \theta, \Lambda, \mathcal{F}, p)$ hold.

Proof. Let $\widehat{A}=\left(\bar{A}_{k}\right) \in \mathcal{C}^{I}(\widehat{A}, \theta, \Lambda, \mathcal{G}, p)$ be any element.

Then, there exists some interval number $\bar{A}$ such that the set

$$
\left\{r \in \mathbf{N}: \frac{1}{h_{r}} \sum_{k \in I_{r}} f_{k}\left(\left\|\Lambda_{k}\left(\bar{A}_{k}\right)-\bar{A}\right\|\right)^{p_{k}} \geq \epsilon\right\} \in I .
$$

Since, each $f_{k}$ is modulus function for all $k \in \mathbf{N}$, we have

$$
\begin{gathered}
\frac{1}{h_{r}} \sum_{k \in I_{r}} f_{k}\left(\left\|\Lambda_{k}\left(\bar{A}_{k}\right)\right\|\right)^{p_{k}} \\
=\frac{1}{h_{r}} \sum_{k \in I_{r}} f_{k}\left(\left\|\Lambda_{k}\left(\bar{A}_{k}\right)-\bar{A}+\bar{A}\right\|\right)^{p_{k}} \\
\leq \frac{1}{h_{r}} \sum_{k \in I_{r}} f_{k}\left(\left\|\Lambda_{k}\left(\bar{A}_{k}\right)-\bar{A}\right\|\right)^{p_{k}}+\frac{1}{h_{r}} \sum_{k \in I_{r}} f_{k}(\|\bar{A}\|)^{p_{k}} .
\end{gathered}
$$

Taking supremum over $r$ on both sides, we get $\widehat{A}=\left(\bar{A}_{k}\right) \in \ell_{\infty}^{I}(\widehat{A}, \theta, \Lambda, \mathcal{F}, p)$. The inclusion $\mathcal{C}_{0}^{I}(\mathcal{A}, \theta, \Lambda, \mathcal{F}, p) \subset \mathcal{C}^{I}(\mathcal{A}, \theta, \Lambda, \mathcal{F}, p)$ is obvious.

Hence $\mathcal{C}_{0}^{I}(\widehat{\mathcal{A}}, \theta, \Lambda, \mathcal{F}, p) \subset \mathcal{C}^{I}(\widehat{\mathcal{A}}, \theta, \Lambda, \mathcal{F}, p) \subset \ell_{\infty}^{I}(\widehat{\mathcal{A}}, \theta, \Lambda, \mathcal{F}, p)$.

Theorem 2.9. The spaces $\mathcal{C}_{0}^{I}(\mathcal{A}, \theta, \Lambda, \mathcal{F}, p)$ and $\mathcal{C}^{I}(\mathcal{A}, \theta, \Lambda, \mathcal{F}, p)$ are sequence algebra.

Proof. Let $\mathbb{A}=\left(\bar{A}_{k}\right), \widetilde{\mathcal{F}}=\left(\bar{B}_{k}\right) \in \mathcal{C}_{0}^{I}(\widetilde{\mathcal{A}}, \theta, \Lambda, \mathcal{F}, p)$. Then, the sets

$$
\left\{r \in \mathbf{N}: \frac{1}{h_{r}} \sum_{k \in I_{r}} f_{k}\left(\left\|\Lambda_{k}\left(\bar{A}_{k}\right)\right\|\right)^{p_{k}} \geq \epsilon\right\} \in I
$$


and

$$
\left\{r \in \mathbf{N}: \frac{1}{h_{r}} \sum_{k \in I_{r}} f_{k}\left(\left\|\Lambda_{k}\left(\bar{B}_{k}\right)\right\|\right)^{p_{k}} \geq \epsilon\right\} \in I .
$$

Therefore, from (28) and (29), we have

$$
\left\{r \in \mathbf{N}: \frac{1}{h_{r}} \sum_{k \in I_{r}} f_{k}\left(\left\|\Lambda_{k}\left(\bar{A}_{k} \cdot \bar{B}_{k}\right)\right\|\right)^{p_{k}} \geq \epsilon\right\} \in I .
$$

Thus, $\widehat{A} \widehat{\mathcal{B}} \in \mathcal{C}_{0}^{I}(\widehat{A}, \theta, \Lambda, \mathcal{F}, p)$. Hence, $\mathcal{C}_{0}^{I}(\widehat{A}, \theta, \Lambda, \mathcal{F}, p)$ is a sequence algebra. Similarly, we can prove that $\mathcal{C}^{I}(\widehat{\mathcal{A}}, \theta, \Lambda, \mathcal{F}, p)$ is a sequence algebra.

\section{References}

[1] A. Esi, A new class of interval numbers, Journal of Qafqaz University, Mathematics and Computer Science, pp. 98-102, (2012).

[2] A. Esi, Lacunary sequence spaces of interval numbers, Thai Journal of Mathemaatics, 10 (2), pp. 445-451, (2012).

[3] A. Esi, Double lacunary sequence spaces of double sequence of interval numbers, Proyecciones Journal of Mathematics, 31 (1), pp. 297-306, (2012).

[4] A.Esi, Strongly almost -convergence and statistically almost convergence of interval numbers, Scientia Magna, 7 (2), pp. 117-122, (2011).

[5] A.Esi, Statistical and lacunary statistical convergence of interval numbers in topological groups, Acta Scientarium Technology, (2014)(Bas?lacak).

[6] A.Esi and N.Braha, On asymptotically -statistical equivalent sequences of interval numbers, Acta Scientarium Technology, 35 (3), pp. 515-520, (2013).

[7] A.Esi and A.Esi, Asymptotically lacunary statistically equivalent sequences of interval numbers, International Journal of Mathematics and Its Applications, 1 (1), pp. 43-48, (2013). 
[8] Ayhan, Esi and Hazarika, B., Some I-convergent of double $\Lambda$-interval sequences defined by Orlicz function., Global, J. of Mathematical Analysis, 1 (3), pp. 110-116, (2013).

[9] Ayhan, Esi., $\lambda$-Sequence Spaces of Interval Numbers, Appl. Math. Inf. Sci. 8, No. 3, pp. 1099-1102, (2014).

[10] Buck, R. C., Generalized asymptotic density,Amer., J. Math. 75, pp. 335-346 (1953).

[11] Chiao, K. P., Fundamental properties of interval vector max-norm, Tamsui Oxford Journal of Mathematical Sciences, 18(2), pp. 219-233, (2002).

[12] Dwyer, P. S., Linear Computation, New York, Wiley, (1951).

[13] Fast, H., Sur la convergence statistique, Colloq. Math. 2, pp. 241-244, (1951).

[14] Freedman, A. R.,Sember,J.J.and Raphael,M.,Some Cesaro type summability spaces, Proc. London Math. Soc., 37, pp. 508-520, (1978).

[15] Fridy, J. A., On statistical convergence, Analysis, 5, pp. 301-313, (1985).

[16] Khan, V. A., Mohd Shafiq and Ebadullah, K., On paranorm Iconvergent sequence spaces of interval numbers, J. of Nonlinear Analysis and Optimisation (Theory and Application), Vol. 5. No. 1, pp. 103-114, (2014).

[17] Khan, V. A., Ahyan Esi and Mohd Shafiq, On paranorm BV $V_{\sigma}$ convergent sequence spaces defined by an Orlicz function, Global Journal of mathematical Analysis, 2 (2), pp. 28-43, (2014).

[18] Kolk, E., On strong boundedness and summability with respect to a sequence of moduli, Acta Comment.Univ.Tartu., 960, pp. 41-50, (1993).

[19] Kolk, E., Inclusion theorems for some sequence spaces defined by a sequence of modulii, Acta Comment.Univ. Tartu., 970, pp. 65-72, (1994).

[20] Kostyrko, P., Mačaj, M.,and Šalát, T., Statistical convergence and Iconvergence.Real Analysis Exchange.

[21] Kostyrko, P., Šalát, T. and Wilczyński, W., I-convergence,Raal Analysis Analysis Exchange. 26(2), pp. 669-686 (2000). 
[22] Moore, R. E., Automatic Error Analysis in Digital Computation, LSMD-48421, Lockheed Missiles and Space Company, (1959).

[23] Moore, R. E. and Yang,C. T., Interval Analysis I, LMSD-285875, Lockheed Missiles and Space Company, Palo Alto, Calif., (1959).

[24] Mursaleen, M. and Abdullah K. Noman, On the Spaces of $\lambda$ Convergent and Bounded Sequences, Thai Journal of Mathematics Volume 8, Number 2, pp. 311-329, (2010).

[25] Mursaleen, M. and Sunil K. Sharma, Spaces of Ideal Convergent sequences,Volume 2014, Article ID 134534, 6 pages http://dx.doi. org $/ 10.1155 / 2014 / 134534$.

[26] Nakano,H., Concave modulars. J. Math Soc. Japan, 5, pp. 29-49, (1953).

[27] Ruckle, W. H., On perfect Symmetric BK-spaces., Math. Ann. 175, pp. 121-126, (1968).

[28] Ruckle, W. H., Symmetric coordinate spaces and symmetric bases, Canad. J. Math. 19, pp. 828-838, (1967).

[29] Ruckle, W. H., FK-spaces in which the sequence of coordinate vectors is bounded, Canad. J. Math. 25 (5), pp. 973-975, (1973).

[30] Šalát, T., On statistical convergent sequences of real numbers,Math, Slovaca 30, (1980).

[31] Šalát, T., Tripathy, B. C. and Ziman, M., On some properties of Iconvergence Tatra Mt. Math. Publ. 28, pp. 279-286, (2004).

[32] Šalát, T., Tripathy, B. C.and Ziman, M., On I-convergence field., Ital. J. Pure Appl. Math. 17, pp. 45-54, (2005).

[33] Schoenberg, I. J., The integrability of certain functions and related summability methods, Amer. Math. Monthly, 66, pp. 361-375, (1959).

[34] Sengönül, M. and Eryilmaz, A., On the Sequence Spaces of Interval Numbers, Thai J. of Mathematics, Volume 8, No. 3, pp. 503-510, (2010).

[35] Tripathy, B. C., On statistical convergence, Proc.Estonian Acad. Sci. Phy. Math. Analysis, pp. 299-303, (1998). 
[36] Tripathy, B. C. and Hazarika, B., Paranorm I-convergent sequence spaces, Math. Slovaca 59 (4), pp. 485-494, (2009).

\section{Mohd Shafiq}

Department of Mathematics

Govt. Degree College,Poonch

Jammu and Kashmir-185101

INDIA

e-mail : shafiqmaths7@gmail.com

and

\section{Ayhan Esi}

Adiyaman University

Departmet of Mathematics

Adiyaman-02040

TURKEY

e-mail: aesi237@hotmail.com 